\title{
Estratégias de educação alimentar e nutricional na Atenção Primária à Saúde: uma revisão de literatura
}

\author{
Food and nutrition education strategies in Primary Health Care: a \\ literature review
}

Camila de Jesus França1, Vivian Carla Honorato dos Santos de Carvalho ${ }^{2}$

\begin{abstract}
RESUMO O presente trabalho objetivou revisar de forma sistemática a produção científica sobre intervenções de educação alimentar e nutricional com indivíduos adultos no campo da Atenção Primária à Saúde no Brasil, no período de 2006 a 2016. Foram selecionados 11 artigos que responderam aos critérios de elegibilidade. Observou-se uma descrição breve das bases teórico-metodológicas, limitando a reprodução das intervenções. As práticas educativas desenvolvidas possuem pouco enfoque na promoção da saúde e uma forte tendência metodológica clássica, sendo necessários estudos qualitativos, com a percepção da autonomia, e que ampliem o uso de metodologias ativas nos processos de intervenção.
\end{abstract}

PALAVRAS-CHAVE Educação alimentar e nutricional. Atenção Primária à Saúde. Serviços de saúde.

ABSTRACT This study aimed to systematically review the scientific production on interventions of food and nutritional education with adults individuals in the area of Primary Health Care in Brazil, in the period from 2006 to 2016.11 articles were selected which responded to the egibililty criteria. A brief description of the theoretical-methodological bases was observed, limiting the production of interventions. The educational practices developed have little focus on health promotion and strong classical methodological tendency. It is necessary to perform qualitative studies with perception of autonomy and that will increase the use of active methodologies in the intervention processes.

KEYWORDS Food and nutrition education. Primary Health Care. Health service.

1Universidade Federal da Bahia (UFBA), Instituto Multidisciplinar em Saúde (IMS) - Vitória da Conquista (BA), Brasil. mylah12@yahoo.com.br

2 Universidade Federal da Bahia (UFBA), Instituto Multidisciplinar em Saúde (IMS) - Vitória da Conquista (BA), Brasil. vihonorato@hotmail.com 


\section{Introdução}

A Educação Alimentar e Nutricional (EAN) se conceitua como um objeto de ação multiprofissional, intersetorial e transdisciplinar, em que o conhecimento e o aprendizado, contínuo e permanente, propõem-se a desenvolver a autonomia e a voluntariedade ante os hábitos alimentares saudáveis, fazendo o uso de recursos e abordagens educacionais ativas e problematizadoras (BRASIL, 2012B). Dessa forma, a EAN protagoniza importante eixo na promoção de hábitos alimentares saudáveis, sendo uma estratégia indispensável dentro das políticas públicas em alimentação e nutrição (BOOG, 2013).

A educação nutricional vem sofrendo importantes modificações no decorrer do tempo. Percebe-se que, na década de 1940, as estratégias se baseavam em ensinar a alimentação dita como correta, com um olhar puramente biológico, sendo prevalente o binômio alimentação-educação. A partir da década de 1970, ocorre um redirecionamento das políticas de alimentação e nutrição, em que a renda é considerada a principal dificuldade em se obter uma alimentação saudável, e não mais a educação. Além disso, a educação nutricional passa a ser vista como uma prática não ética e desnecessária, com poucos estudos sendo desenvolvidos, ficando por duas décadas praticamente ausente entre os programas de saúde pública (BOOG, 1997; GREENWOOD; FONSECA, 2016). A partir de 1990, surgem então pesquisas relacionadas com a saúde que apontavam a influência dos hábitos alimentares como causas para o aumento das doenças crônicas não transmissíveis, sendo surpreendidos com obesidade como um grande problema de saúde pública, posicionando a EAN como uma medida imprescindível perante a proteção e a construção de hábitos saudáveis no enfrentamento dos problemas alimentares e nutricionais do contexto atual (BRASIL, 2012A).
Nota-se, a partir de então, como a EAN se torna pertinente no que tange às políticas públicas de alimentação e nutrição devido à necessidade de ampliar a discussão sobre as possibilidades, limites e o modo como a EAN é realizada, resultando em ações governamentais, especialmente as desenvolvidas pelos Ministérios do Desenvolvimento Social e de Combate à Fome, levando à construção do Marco de Referência de Educação Alimentar e Nutricional para as Políticas Públicas, que reflete uma importante etapa de valorização dessas ações (GREENWOOD; FONSECA, 2016).

A Atenção Primária à Saúde (APS) é reconhecida como uma estratégia essencial no combate à disparidade da assistência à saúde no Brasil, representando o contato preferencial dos usuários com os sistemas de saúde. No País, o termo APS é considerado equivalente ao termo Atenção Básica $(A B)$, sendo os termos utilizados nas políticas e literatura nacional. O desenvolvimento das ações na APS parte de técnicas educativas que possam intervir no processo de saúde-doença da população. Nesse sentido, a alimentação e a nutrição fazem parte das condições básicas para a promoção e proteção à saúde, sendo que as estratégias de EAN representam papel fundamental no contexto da $\mathrm{AB}$ e, em especial, na Estratégia Saúde da Família (ESF), que surge para reorganizar a rede de atenção a fim de aproximar o trabalho educativo na comunidade, ampliando o seu campo de intervenções (ALENCAR ET AL., 2016; BRASIL, 2012A; JAIME ET AL., 2011; OLIVEIRA; PEREIRA, 2013).

Segundo Pimentel et al. (2011), a maior demanda de atendimentos na ESF é representada pela população adulta. Isso pode estar relacionado com a alta frequência de doenças crônicas não transmissíveis que acometem essa faixa etária, além das mudanças demográficas que vêm ocorrendo no Brasil com a diminuição da fecundidade e da mortalidade infantil e com o aumento da expectativa de vida. Além 
disso, ressalta-se a importância de compreender as intervenções aplicadas na população adulta nos serviços de saúde da atenção primária e como tal fato é relevante para a promoção da saúde desse grupo específico.

Mesmo diante dos avanços e acúmulos de conhecimento sobre EAN e sua crescente valorização, sobretudo nas políticas públicas em alimentação e nutrição, seu âmbito de atuação não está distintamente definido, dispondo de diferentes entendimentos quanto a sua abordagem prática e conceitual, bem como um consenso das dificuldades de desenvolver intervenções em decorrência da baixa existência de referenciais teóricos, metodológicos e operacionais, havendo escassas referências quanto aos elementos que presidem sua prática (SANTOS, 2012).

Diante do exposto, evidencia-se a importância de estudos que favoreçam a promoção de práticas no âmbito da EAN verdadeiramente efetivas. Desse modo, o presente estudo teve como objetivo revisar de forma sistematizada a produção científica brasileira sobre intervenções de EAN com indivíduos adultos no campo da APS no Brasil, no período de 2006 a 2016.

\section{Metodologia}

Esse estudo foi desenvolvido segundo critérios de revisão sistemática, em que inicialmente produziu-se um protocolo para conduzir a revisão. De acordo com esse protocolo, foram selecionados todos os estudos cujos títulos ou resumos mencionassem a apresentação de dados originais sobre EAN e práticas de intervenção em adultos atendidos na APS no Brasil.

Para nortear a pesquisa, foi definida como questão de pesquisa: Que intervenções estão sendo utilizadas como instrumento para EAN com usuários adultos de serviços de saúde na atenção primária no Brasil?

\section{Estratégias de busca}

Utilizou-se como metodologia a busca bibliográfica de artigos nas bases de dados Scientific Electronic Library Online (SciELO), Biblioteca Virtual em Saúde e no Periódico Capes (Coordenação de Aperfeiçoamento de Pessoal de Nível Superior), a fim de reconhecer artigos científicos publicados entre os anos de 2006 e 2016. A busca teve como descritores os termos 'educação alimentar e nutricional' $A N D$ 'centros de saúde', $A N D$ 'estratégia saúde da família', $A N D$ 'atenção primária à saúde' $A N D$ 'serviços de saúde', bem como seus correspondentes em inglês, 'food and nutrition education' $A N D$ 'health centers', $A N D$ 'family health strategy' AND 'primary health care', AND 'health services'. Os artigos foram pré-selecionados pelos títulos em que deveriam conter referência à EAN e/ou intervenção nutricional na atenção primária seguido da leitura dos resumos de livre acesso.

Foi definida APS como descritor, visto esse ter um reconhecimento internacional e ser sinônimo do descritor 'atenção básica'.

\section{Critérios de elegibilidade}

Todo o material obtido pelo sistema de busca referido foi submetido aos seguintes critérios de inclusão: ser artigos originais, de acesso livre, ser publicado nos periódicos de 2006 a 2016, propor intervenções e/ou avaliações de atividades abrangendo a educação nutricional na APS, participantes com idade maior que 20 anos, independentemente do sexo, etnia e nível socioeconômico, e artigos desenvolvidos no Brasil.

Foram utilizados como critérios de exclusão: ser artigos curtos, teses, trabalhos que não foram publicados no período considerado e/ou que não estivessem inseridos nas referidas bases de dados, estudos sem acesso livre, além dos artigos que não aplicaram 
algum tipo de intervenção nesse âmbito, ou seja, não realizaram ou avaliaram atividades de educação nutricional na atenção primária ou foram desenvolvidos com crianças, adolescentes, gestantes e lactantes e/ou que não foram desenvolvidos no Brasil.

Foram incluídas publicações em português e inglês, sendo rejeitados os artigos repetidos nas bases de dados. A seleção dos estudos levantados foi categorizada de acordo com o tipo de estudo, objetivos, público-alvo, local da pesquisa e ano de publicação do artigo, bem como as revistas em que estavam relacionadas, metodologia aplicada e seus principais resultados.

\section{Avaliação dos artigos segundo a classificação dos periódicos}

Com o intuito de avaliar as revistas a que os estudos estavam veiculados, utilizou-se a classificação de periódicos do Qualis/Capes, tendo como referência a área de avaliação de nutrição e saúde coletiva, como pode ser visto no estudo de Ramos, Santos e Reis (2013) que, dos artigos selecionados, fundamentou-se a categorização de periódicos no Qualis/ Capes, tendo como parâmetro a área de avaliação de nutrição, sendo que as revistas que possuem classificação Al representavam o maior indicativo de qualidade dos veículos de divulgação, e C, o menor indicador.

\section{Resultados}

A busca foi realizada, segundo estratégia predefinida, resultando em um achado de 2.506 estudos, referenciados em três bases dados: SciELO, Biblioteca Virtual em Saúde e o Periódico Capes, por meio de descritores em inglês e português, publicados entre os anos de 2006 e 2016, sendo encontrados: 200 estudos na base SciELO, 1.364 estudos na base Biblioteca Virtual em Saúde e 942 estudos na base Periódico Capes. Deste total de estudos, foram selecionados 11 trabalhos sobre intervenções em EAN na APS em indivíduos adultos com idade acima de 20 anos. A figura 1 apresenta o fluxograma da seleção dos artigos. 
Figura 1. Fluxograma das etapas de seleção dos artigos revisados

\begin{tabular}{|c|c|}
\hline $\begin{array}{l}\text { Artigos obtidos na pesquisa } \\
\text { bibliográfica: } n=2.506\end{array}$ & $\begin{array}{l}\text { Artigos excluídos pela leitura dos } \\
\text { títulos: } n=2.431 \\
\text { Texto que não foi possível acesso: } \\
\qquad n=36\end{array}$ \\
\hline $\begin{array}{l}\text { Artigos selecionados pelo título } \\
\text { para leitura de resumo: } n=39\end{array}$ & \multirow[b]{2}{*}{$\begin{array}{l}\text { Artigos excluídos após leitura de resumo } \\
\text { com justificativa: } n=24 \\
\text { A intervenção não era em Atenção } \\
\text { Primária à Saúde: } n=11 \\
\text { O trabalho não estava no formato } \\
\text { de artigo: } n=7 \\
\text { Intervenções realizadas em outros } \\
\text { países: } n=4 \\
\text { Não era intervenção: } n=2\end{array}$} \\
\hline | & \\
\hline & Artigos duplicados: $n=2$ \\
\hline $\begin{array}{l}\text { Artigos em texto completo } \\
\text { avaliados para elegibilidade: } \\
n=13\end{array}$ & \\
\hline$\downarrow$ & \multirow[t]{2}{*}{$\begin{array}{l}\text { Número de artigos em texto completo } \\
\text { excluído: } n=2 \\
\text { Trabalho apresentava metodologia } \\
\text { não adequada: } n=1 \\
\text { Trabalho duplicado: } n=1\end{array}$} \\
\hline Estudos selecionados: $n=11$ & \\
\hline
\end{tabular}

Para a identificação das revistas na qual os artigos foram publicados utilizou-se o sistema de classificação Qualis/Capes. Dos artigos selecionados, 45,4\% apresentaram
Qualis B2, 36,4\% com Qualis B3 e 18,2\% dos artigos com Qualis B4 e B5, não havendo revistas que possuíssem classificação Ale C, como está categorizado no quadro 1. 


\begin{tabular}{lll}
\hline Quadro 1. Classificação dos estudos segundo o Qualis & \\
\hline Estudo & Revista & Qualis \\
\hline Deus et al., 2015 & Revista Ciência \& Saúde Coletiva & B2 \\
Mendonça; Lopes, 2012 & Revista da Escola de Enfermagem da USP & B3 \\
Ribeiro et al., 2012 & Revista de Nutrição & B3 \\
Gomes et al., 2013 & Revista Brasileira em Promoção da Saúde & B5 \\
Anunciação et al., 2012 & Revista Baiana de Saúde Pública & B2 \\
Machado et al., 2016 & Revista Ciência \& Saúde Coletiva & B3 \\
Gandra et al., 2011 & Revista Brasileira em Promoção da Saúde & B2 \\
Teixeira et al., 2013 & Revista Ciência\& Saúde Coletiva & B3 \\
Silva et al., 2012 & Revista de APS - Atenção Primária à Saúde & B2 \\
Silva et al., 2013 & Revista de Nutrição & B4 \\
Silva; Quintão, 2015 & Revista Brasileira de Obesidade, Nutrição e Emagrecimento &
\end{tabular}

USP (Universidade de São Paulo); APS (Atenção Primária à Saúde).

Os estudos que elaboraram alguma intervenção com base na educação nutricional realizada dentro da APS no Brasil, e que avaliaram os resultados após a execução, foram caracterizados no quadro 2 quanto: ao autor, ao ano de publicação, ao local do estudo, ao tipo de estudo e à revista em que foram publicados. Observa-se que, mesmo os artigos sendo refinados no período de 2006 a 2016, os estudos encontrados iniciam-se com o ano de publicação em 2011, não sendo encontrados artigos anteriores a esse período. Quanto ao local em que os estudos foram executados, nota-se que $81,8 \%$ dos trabalhos foram desenvolvidos no estado de Minas Gerais, 9,1\% no estado de São Paulo e 9,1\% no estado de Sergipe, demonstrando uma predominância na região Sudeste.

Quadro 2. Estudos de intervenção em educação alimentar e nutricional em adultos atendidos na APS no Brasil quanto ao local de realização, tipo de estudo e revista na qual foram publicados no País no período de 2006 a 2016

\begin{tabular}{|c|c|c|c|c|}
\hline Estudo & Ano & Local do Estudo & Tipo de Estudo & Revista \\
\hline Deus et al. & 2015 & Belo Horizonte (Minas Gerais) & Intervenção não aleatória & Revista Ciência \& Saúde Coletiva \\
\hline Mendonça; Lopes & 2012 & $\begin{array}{l}\text { Região leste de Belo Horizonte } \\
\text { (Minas Gerais) }\end{array}$ & Intervenção Quase-experimental & Revista da Escola de Enfermagem da USP \\
\hline Ribeiro et al. & 2012 & Porto Firme (Minas Gerais) & $\begin{array}{l}\text { Intervenção de abordagem qualiquan- } \\
\text { titativa }\end{array}$ & Revista de Nutrição \\
\hline Gomes et al. & 2013 & Diadema (São Paulo) & $\begin{array}{l}\text { Longitudinal e natureza quantitativa, } \\
\text { com intervenção educativa }\end{array}$ & Revista Brasileira em Promoção da Saúde \\
\hline Anunciação et al. & 2012 & Ouro Preto (Minas Gerais) & Estudo de intervenção & Revista Baiana de Saúde Pública \\
\hline Machado et al. & 2016 & $\begin{array}{l}\text { Área urbana de um município } \\
\text { de pequeno porte }\end{array}$ & $\begin{array}{l}\text { Intervenção longitudinal, comparativo, } \\
\text { do tipo ensaio comunitário }\end{array}$ & Revista Ciência \& Saúde Coletiva \\
\hline Gandra et al. & 2011 & Diamantina (Minas Gerais) & $\begin{array}{l}\text { Estudo de intervenção do tipo antes e } \\
\text { depois }\end{array}$ & Revista Brasileira em Promoção da Saúde \\
\hline Teixeira et al. & 2013 & Aracaju (Sergipe) & Estudo do tipo pré-teste/pós-teste & Revista Ciência\& Saúde Coletiva \\
\hline
\end{tabular}


Quadro 2. (cont.)

\begin{tabular}{lllll}
\hline Silva et al. & 2012 & Belo Horizonte (Minas Gerais) & Estudo de intervenção & Revista de APS - Atenção Primária à Saúde \\
Silva et al. & 2013 & Belo Horizonte (Minas Gerais) & Intervenção aberta e não controlada & Revista de Nutrição \\
Silva; Quintão & 2015 & Muriaé (Minas Gerais) & $\begin{array}{l}\text { Intervenção do tipo longitudinal, qualita- } \\
\text { tiva e quantitativa }\end{array}$ & $\begin{array}{l}\text { Revista Brasileira de Obesidade, Nutrição e } \\
\text { Emagrecimento }\end{array}$ \\
\hline
\end{tabular}

USP (Universidade de São Paulo); APS (Atenção Primária à Saúde).

O quadro 3 discrimina os artigos quanto: aos objetivos do estudo, à metodologia desenvolvida e aos principais resultados obtidos. Sobre os objetivos, observou-se que $36,4 \%$ dos estudos tinha por objetivo avaliar uma intervenção em EAN sobre um grupo específico que estivesse vinculado à APS, mais precisamente sobre o público que frequentava a Academia da Saúde, como pode ser visto em 4 dos 11 estudos selecionados, associando a prática de exercício físico com a intervenção em EAN. Os outros artigos dispunham de intervenções sobre uma população específica com alguma Doença Crônica Não Transmissível (DCNT), como hipertensão arterial sistêmica, diabetes mellitus e obesidade, sem associação com a prática de exercícios físicos apenas com o desenvolvimento de atividades em EAN.

Quadro 3. Categorização dos artigos que apresentam estudos de intervenção em educação alimentar e nutricional em adultos atendidos na APS no Brasil quanto aos objetivos, metodologia e principais resultados, publicados no Brasil no período de 2006 a 2016

\begin{tabular}{|c|c|c|c|c|c|}
\hline \multirow{2}{*}{ Objetivos } & \multicolumn{4}{|c|}{ Metodologia } & \multirow{2}{*}{ Principais resultados } \\
\hline & Público-alvo & Período & Intervenção & Avaliação da intervenção & \\
\hline \multicolumn{6}{|c|}{ Deus et al., 2015} \\
\hline $\begin{array}{l}\text { Avaliar o impacto de inter- } \\
\text { venção nutricional associa- } \\
\text { da à prática de exercícios } \\
\text { físicos sobre o perfil ali- } \\
\text { mentar e antropométrico } \\
\text { de usuárias do Programa } \\
\text { Academia da Saúde no } \\
\text { município de Belo Horizon- } \\
\text { te (MG). }\end{array}$ & $\begin{array}{l}124 \text { mulheres } \\
\text { participantes do } \\
\text { Programa Acade- } \\
\text { mia da Saúde de } \\
\text { Belo Horizonte, } \\
\text { MG. }\end{array}$ & 11 meses & $\begin{array}{l}\text { Participantes expostas à } \\
\text { intervenção nutricional com } \\
\text { o uso de ilustrações por } \\
\text { meio de materiais educati- } \\
\text { vos e lúdicos, como réplicas, } \\
\text { fotos de alimentos e medi- } \\
\text { das caseiras, além de jogos } \\
\text { educativos e teatro, bem } \\
\text { como da atividade física } \\
\text { pelo educador físico. }\end{array}$ & $\begin{array}{l}\text { Antropometria e indicadores } \\
\text { dietéticos antes da inter- } \\
\text { venção e após } 11 \text { meses de } \\
\text { seu início. }\end{array}$ & $\begin{array}{l}\text { Impacto positivo no perfil } \\
\text { alimentar e antropomé- } \\
\text { trico. }\end{array}$ \\
\hline \multicolumn{6}{|c|}{ Mendonça; Lopes, 2012} \\
\hline $\begin{array}{l}\text { Avaliar o efeito de inter- } \\
\text { venções nutricionais rela- } \\
\text { cionados com os exercícios } \\
\text { físicos regulares e hábitos } \\
\text { alimentares, sobre asmedi- } \\
\text { das físicas de usuários de } \\
\text { um serviço de promoção } \\
\text { da saúde, a Academia da } \\
\text { Saúde de Belo Horizonte - } \\
\text { Minas Gerais, Brasil. }\end{array}$ & $\begin{array}{l}167 \text { usuários que } \\
\text { realizavam exer- } \\
\text { cício físico regular } \\
\text { na academia } \\
\text { desaúde. }\end{array}$ & 7 meses & $\begin{array}{l}\text { A intervenção contou com } \\
\text { a avaliação por meio de } \\
\text { anamnese nutricional, an- } \\
\text { tropometria e aferição da } \\
\text { PA,atendimento nutricional } \\
\text { coletivo e individual e a } \\
\text { prática de exercício físico. }\end{array}$ & $\begin{array}{l}\text { Após a intervenção, houve a } \\
\text { reavaliação antropométrica, } \\
\text { aferição da PA e aplicação } \\
\text { do questionário 'Como está } \\
\text { sua alimentação?'. }\end{array}$ & $\begin{array}{l}\text { Adoção de hábitos ali- } \\
\text { mentares saudáveis e } \\
\text { redução da CC eadequa- } \\
\text { ção da PA sistólica. }\end{array}$ \\
\hline
\end{tabular}


Quadro 3. (cont.)

\begin{tabular}{|c|c|c|c|c|c|}
\hline \multicolumn{6}{|c|}{ Ribeiro et al., 2012} \\
\hline $\begin{array}{l}\text { Analisar a importância, a } \\
\text { efetividade e as limitações } \\
\text { de estratégias de educação } \\
\text { em saúde, relacionadas } \\
\text { com as orientações dieté- } \\
\text { ticas, no enfrentamento da } \\
\text { problemática da adesão } \\
\text { ao tratamento da HAS, no } \\
\text { contexto do Programa de } \\
\text { Saúde da Família. }\end{array}$ & $\begin{array}{l}27 \text { mulheres entre } \\
45 \text { e } 60 \text { anos, } \\
\text { com diagnóstico } \\
\text { confirmado de } \\
\text { HAS. }\end{array}$ & 5 meses & $\begin{array}{l}\text { As participantes foram alo- } \\
\text { cadas em dois grupos. Um } \\
\text { grupo com oficinas mensais } \\
\text { com vídeos, dinâmicas e } \\
\text { palestras, e outro grupo que } \\
\text { participou das oficinas e } \\
\text { recebeu orientação individu- } \\
\text { alizada e familiar no domicí- } \\
\text { lio por nutricionista. }\end{array}$ & $\begin{array}{l}\text { Foram realizadas entrevis- } \\
\text { tas e GF para conhecer a } \\
\text { percepção das participantes } \\
\text { sobre as atividades educa- } \\
\text { tivas e as mudanças nos } \\
\text { hábitos alimentares. Antes } \\
\text { e após a intervenção, houve } \\
\text { a avaliação antropométrica, } \\
\text { aferição da PA, dos níveis de } \\
\text { glicemia ecolesterol. }\end{array}$ & $\begin{array}{l}\text { O grupo que recebeu as } \\
\text { VD apresentou melhorias } \\
\text { na avaliação clínica, com } \\
\text { redução dos parâmetros } \\
\text { de IMC, da PA sistólica } \\
\text { e glicemia, enquanto o } \\
\text { grupo que não recebeu } \\
\text { orientações domiciliares } \\
\text { teve redução apenas da } \\
\text { CC. }\end{array}$ \\
\hline \multicolumn{6}{|c|}{ Gomes et al., 2013} \\
\hline $\begin{array}{l}\text { Avaliar o impacto de um } \\
\text { programa de educação } \\
\text { nutricional sobre o estado } \\
\text { nutricional e conhecimento } \\
\text { alimentar de pacientes } \\
\text { com excesso de peso. }\end{array}$ & $\begin{array}{l}63 \text { pacientes de } \\
\text { ambos os sexos, } \\
\text { adultos e idosos, } \\
\text { alfabetizados, } \\
\text { frequentadores } \\
\text { de um grupo de } \\
\text { educação alimen- } \\
\text { tar para pessoas } \\
\text { com excesso de } \\
\text { peso. }\end{array}$ & 7semanas & $\begin{array}{l}\text { Desenvolveram a educação } \\
\text { nutricional por meio de } \\
\text { aulas expositivas e conver- } \\
\text { sas em grupo, discutindo } \\
\text { diferentes temas referentes } \\
\text { à alimentação saudável e } \\
\text { aplicação de questionários } \\
\text { para avaliar o nível de co- } \\
\text { nhecimento dos pacientes. }\end{array}$ & $\begin{array}{l}\text { Foi realizado com aplicação } \\
\text { de questionários no inicio e } \\
\text { no final de cada encontro, } \\
\text { com o intuito de avaliar } \\
\text { a compreensão de cada } \\
\text { participante em relação ao } \\
\text { assunto desenvolvido na } \\
\text { educação nutricional e a } \\
\text { avaliação antropométrica } \\
\text { pré e pós-intervenção. }\end{array}$ & $\begin{array}{l}\text { Redução das medidas } \\
\text { antropométricas, com } \\
\text { redução de peso, evolu- } \\
\text { ção no estado nutricional } \\
\text { e melhoria no conheci- } \\
\text { mento sobre alimenta- } \\
\text { ção saudável. }\end{array}$ \\
\hline \multicolumn{6}{|c|}{ Anunciação et al., 2012} \\
\hline $\begin{array}{l}\text { Avaliar o conhecimento } \\
\text { sobre alimentação entre } \\
\text { pacientes com diagnóstico } \\
\text { de DM tipo } 2 \text { atendidos na } \\
\text { UBS Bauxita, bem como } \\
\text { avaliar o perfil nutricional } \\
\text { dessas pessoas e sua } \\
\text { relação com o nível de } \\
\text { conhecimento acerca da } \\
\text { doença. }\end{array}$ & $\begin{array}{l}33 \text { usuários, } \\
\text { adultos e idosos } \\
\text { de ambos os } \\
\text { sexos atendidos } \\
\text { na UBS Bauxita, } \\
\text { com diagnóstico } \\
\text { de DM tipo } 2 \text {. }\end{array}$ & $\begin{array}{l}\text { Out. e } \\
\text { nov. de } \\
2010\end{array}$ & $\begin{array}{l}\text { A educação nutricional se } \\
\text { deu mediante avaliação an- } \\
\text { tropométrica e aplicação de } \\
\text { formulário para obtenção de } \\
\text { dados sobre conhecimento } \\
\text { em alimentação e fatores } \\
\text { que possam interferir na } \\
\text { sua realização. Logo após, } \\
\text { foi realizada uma dinâmica } \\
\text { de grupo intitulada 'Bingo } \\
\text { Nutricional'. }\end{array}$ & $\begin{array}{l}\text { Entre o } 70 \text { e } 20 \text { dia após } \\
\text { a realização da dinâmica } \\
\text { educativa, foram realizadas } \\
\text { visitas domiciliares para } \\
\text { uma nova aplicação do teste } \\
\text { com o propósito de avaliar a } \\
\text { eficácia de tal atividade. }\end{array}$ & $\begin{array}{l}\text { Não houve diferença entre } \\
\text { tempo de diagnóstico } \\
\text { da doença e o nível de } \\
\text { conhecimento acerca } \\
\text { da alimentação. Entre } \\
\text { os usuários de insulina, } \\
\text { houve melhor nível de } \\
\text { conhecimento do que } \\
\text { aqueles que fazem uso do } \\
\text { medicamento oral. }\end{array}$ \\
\hline \multicolumn{6}{|c|}{ Gandra et al, 2011} \\
\hline $\begin{array}{l}\text { Avaliar o efeito de um pro- } \\
\text { grama de educação no co- } \\
\text { nhecimento e nas atitudes } \\
\text { sobre o DM de pacientes } \\
\text { diabéticos acompanhados } \\
\text { por duas UBS cidade de } \\
\text { Diamantina (MG). }\end{array}$ & $\begin{array}{l}23 \text { pacientes } \\
\text { diabéticos do tipo } \\
1 \text { ou 2, atendidos } \\
\text { por duas UBS. }\end{array}$ & 12 meses & $\begin{array}{l}\text { As atividades educativas } \\
\text { foram conduzidas por meio } \\
\text { de técnicas instrucionais } \\
\text { como preleção dialogada, } \\
\text { demonstração, teatro, } \\
\text { brainstorming, discussão e } \\
\text { tarefa dirigida. }\end{array}$ & $\begin{array}{l}\text { Foi realizado por meio de } \\
\text { questionário semiestrutura- } \\
\text { do, aplicado antes a após a } \\
\text { intervenção. }\end{array}$ & $\begin{array}{l}\text { Observou-se uma me- } \\
\text { Ihora no nível de conheci- } \\
\text { mento dos participantes } \\
\text { após o trabalho educativo. }\end{array}$ \\
\hline \multicolumn{6}{|c|}{ Teixeira et al., 2013} \\
\hline $\begin{array}{l}\text { Avaliar a eficácia de dois } \\
\text { métodos de intervenção } \\
\text { nutricional educativa entre } \\
\text { mulheres praticantes de } \\
\text { atividade física regular } \\
\text { visando à modificação e à } \\
\text { adoção de práticas alimen- } \\
\text { tares saudáveis. }\end{array}$ & $\begin{array}{l}60 \text { mulheres com } \\
\text { idade entre } 19 \text { e } \\
59 \text { anos partici- } \\
\text { pantes do Progra- } \\
\text { ma Academia da } \\
\text { Cidade, vincula- } \\
\text { das a cinco polos } \\
\text { do programa. }\end{array}$ & 2 meses & $\begin{array}{l}\text { Divididos em dois grupos: } \\
\text { intervenção nutricional } \\
\text { educativa menos intensiva, } \\
\text { realizada em um único } \\
\text { momento com aula dinâmi- } \\
\text { ca, e intervenção nutricional } \\
\text { educativa mais intensiva, } \\
\text { com 7encontros. }\end{array}$ & $\begin{array}{l}\text { Aplicação de questionário } \\
\text { contendo } 12 \text { perguntas de } \\
\text { conhecimento a respeito } \\
\text { do assunto. Avaliaram-se } \\
\text { também as modificações } \\
\text { realizadas na alimentação e } \\
\text { seus principais motivos con- } \\
\text { duzidos ao final da interven- } \\
\text { ção e aferição das medidas } \\
\text { antropométricas. }\end{array}$ & $\begin{array}{l}\text { A intervenção nutricional } \\
\text { educativa mais intensiva } \\
\text { por dois meses e de cará- } \\
\text { ter prático produziu efeito } \\
\text { desejado na mudança de } \\
\text { comportamento alimentar } \\
\text { e na redução do peso cor- } \\
\text { poral no grupo estudado. }\end{array}$ \\
\hline
\end{tabular}




\begin{tabular}{|c|c|c|c|c|c|}
\hline \multicolumn{6}{|c|}{ Silva et al., 2012} \\
\hline $\begin{array}{l}\text { Realizar intervenção nutri- } \\
\text { cional integrada à prática } \\
\text { de atividade física com } \\
\text { usuários hipertensos que } \\
\text { praticam Lian Gong em } \\
\text { uma UBS de Belo Hori- } \\
\text { zonte. }\end{array}$ & $\begin{array}{l}15 \text { usuários dos } \\
\text { serviços da UBS. }\end{array}$ & 3 meses & $\begin{array}{l}\text { A educação nutricional se } \\
\text { deu em6 encontros, com } \\
\text { oficinas educativas e de } \\
\text { como cultivar hortas em } \\
\text { locais pequenos, palestras } \\
\text { dialogadas sobre alimenta- } \\
\text { ção saudável e adequada na } \\
\text { prática de atividade física. }\end{array}$ & $\begin{array}{l}\text { No primeiro e último encon- } \\
\text { tro foi aplicado o questioná- } \\
\text { rio 'Como está sua alimen- } \\
\text { tação?', realizada avaliação } \\
\text { antropométrica e da PA. }\end{array}$ & $\begin{array}{l}\text { Foram verificadas modi- } \\
\text { ficações importantes nos } \\
\text { hábitos alimentares, na } \\
\text { qualidade de vida, com } \\
\text { redução significativa da } \\
\text { CC e da RCQ. }\end{array}$ \\
\hline \multicolumn{6}{|c|}{ Silva et al., 2013} \\
\hline $\begin{array}{l}\text { Avaliar as oficinas, com } \\
\text { foco na aquisição de in- } \\
\text { formações em nutrição e } \\
\text { na percepção de mudança } \\
\text { de hábitos alimentares de } \\
\text { usuários de uma Academia } \\
\text { da Cidade (MG) e avaliar } \\
\text { a viabilidade de executar } \\
\text { essa intervenção nesse } \\
\text { serviço, considerando-se } \\
\text { sua estrutura física e seus } \\
\text { recursos disponíveis. }\end{array}$ & $\begin{array}{l}\text { O número de } \\
\text { participantes } \\
\text { variou entre } 71 \text { e } \\
132 \text { usuários. }\end{array}$ & 4 meses & $\begin{array}{l}\text { As intervenções educacio- } \\
\text { nais foram por meio de ofici- } \\
\text { nas, baseadas nos } 10 \text { passos } \\
\text { para uma alimentação } \\
\text { saudável e o guia alimentar } \\
\text { para a população brasileira, } \\
\text { dinâmica de perguntas e } \\
\text { respostas, uso da pirâmide } \\
\text { alimentar e demonstração } \\
\text { da recomendação de sal, } \\
\text { açúcar, além de contar com } \\
\text { os exercícios físicos em três } \\
\text { dias da semana com dura- } \\
\text { ção de uma hora. }\end{array}$ & $\begin{array}{l}\text { Realizado com aplicação de } \\
\text { dois questionários: um para } \\
\text { a avaliação da aquisição de } \\
\text { informações e outro após } \\
\text { três meses do encerra- } \\
\text { mento das atividades, de } \\
\text { seguimento autopreenchido } \\
\text { acerca de sua percepção } \\
\text { quanto às mudanças ocorri- } \\
\text { das no período. }\end{array}$ & $\begin{array}{l}\text { A intervençãoteve poten- } \\
\text { cial para a aquisição de } \\
\text { informações em nutrição } \\
\text { e a adoção de hábitos } \\
\text { alimentares saudáveis, } \\
\text { como a redução do con- } \\
\text { sumo de óleos, açúcares } \\
\text { e refrigerantes e aumento } \\
\text { do consumo de frutas e } \\
\text { hortaliças. }\end{array}$ \\
\hline \multicolumn{6}{|c|}{ Silva; Quintão, 2015} \\
\hline $\begin{array}{l}\text { Avaliar a eficácia da } \\
\text { intervenção nutricional } \\
\text { nas mudanças do hábito } \\
\text { alimentar e no perfil antro- } \\
\text { pométrico de participantes } \\
\text { dos grupos 'De bem com a } \\
\text { balança' assistidos nas UBS } \\
\text { do município de Muriaé- } \\
\text {-MG. }\end{array}$ & $\begin{array}{l}70 \text { pessoas aten- } \\
\text { didas em quatro } \\
\text { UBS do município } \\
\text { de Muriaé entre } \\
\text { adolescentes, } \\
\text { adultos e idosos } \\
\text { de ambos os } \\
\text { sexos. }\end{array}$ & 4 meses & $\begin{array}{l}\text { As ações de educação } \\
\text { nutricional foram realizada- } \\
\text { sem sete encontros. Após } \\
\text { uma semana, foi entregue a } \\
\text { cada participante um plano } \\
\text { alimentar. Os próximos } \\
\text { encontros foram realizados } \\
\text { quinzenalmente para avalia- } \\
\text { ção antropométrica e parti- } \\
\text { cipação nas palestras sobre } \\
\text { alimentação e saúde. }\end{array}$ & $\begin{array}{l}\text { Aplicação do questionário } \\
\text { com perguntas sobre os } \\
\text { hábitos alimentares e aferi- } \\
\text { ção das medidas antropo- } \\
\text { métricas. }\end{array}$ & $\begin{array}{l}\text { Houve melhora no estado } \\
\text { nutricional dos partici- } \\
\text { pantes, com diminuição } \\
\text { em todos os índices ou } \\
\text { medidas. }\end{array}$ \\
\hline
\end{tabular}

HAS: (Hipertensão Arterial Sistêmica); IMC (Índice de Massa Corporal); CC (Circunferência da Cintura); DM (Diabetes Mellitus); PA (Pressão Arterial); VD (Visita Domiciliar); MG (Minas Gerais); UBS (Unidade Básica de Saúde); RCQ (Razão Circunferência Quadril); GF (Grupo Focal).

Quanto à metodologia desenvolvida nos estudos, nota-se que as intervenções foram realizadas de diferentes formas, apresentando, na maior parte dos trabalhos, mais de uma estratégia desenvolvida em um único grupo, utilizando tanto ferramentas lúdicas como teatro, apresentação de vídeos, fotos, como a avaliação antropométrica e bioquímica, palestras, preenchimento de questionários e até mesmo a realização de visitas domiciliares. O período de duração em que foram desenvolvidas as intervenções variou nos estudos entre 7 semanas e 15 meses.

As avaliações das intervenções se deram por meio da aplicação pré e pós-intervenção, com avaliação antropométrica para a observação quanto ao estado nutricional, além da aplicação de questionário sobre 
conhecimento em nutrição e hábitos alimentares e aferição da pressão arterial. Outros métodos de análise das intervenções também foram utilizados em alguns trabalhos, em menor expressão como o grupo focal relatado em apenas um estudo.

Os estudos selecionados descreveram como resultados a melhora do perfil alimentar, das medidas antropométricas, do estado nutricional dos usuários, bem como dos parâmetros bioquímicos, quanto à glicemia, colesterol, da pressão arterial, além de melhorar a adesão ao tratamento quanto à hipertensão, diabetes e obesidade. Todavia, percebe-se uma fragilidade quando se analisam as questões referentes às estratégias metodológicas de EAN, visto que $63,6 \%$ dos estudos possuem um período de intervenção menor ou igual a 5 meses, não propondo um trabalho contínuo e permanente como preconizado. Além disso, apenas um estudo realizou avaliação qualitativa, na perspectiva de compreender a percepção do participante e as questões inerentes ao empoderamento e autonomia do sujeito sobre os seus hábitos alimentares. Quanto ao uso de abordagens educacionais ativas e problematizadoras, apenas um dos artigos utilizou tais ferramentas para o desenvolvimento das oficinas.

\section{Discussão}

A seleção dos artigos com essa temática partiu da necessidade de conhecer quais intervenções de EAN estão sendo aplicadas para os usuários adultos na atenção primária no Brasil, visto que o baixo número de achados reflete como a EAN ainda precisa ser amplamente discutida, ressaltando a urgência da construção de novas perspectivas para a sua aplicação.

Destaca-se que os estudos analisados iniciaram as suas publicações a partir do ano de 2011, mesmo tendo a pesquisa um intervalo de 10 anos, entre 2006 e 2016. Tal fato pode ser referente à maior ênfase dada à temática nos últimos anos, sendo reforçada com o 'Marco de Referência de Educação Alimentar e Nutricional para as Políticas Públicas', publicado no ano de 2012, contribuindo para o aumento progressivo e significativo do interesse sobre o tema e para a valorização de suas ações.

É indiscutível a crescente importância da EAN atualmente. Entretanto, das publicações encontradas, nenhuma estava veiculada em revista que apresentasse o Qualis/Capes no estrato indicativo de qualidade mais levado. Tal fato nos leva a alguns questionamentos quanto à relevância atribuídos a essa temática no meio cientifico, já que não foram encontradas produções em revistas que estivessem classificadas, na área avaliada, com o maior grau de qualidade que o Qualis/Capes determina. Fato semelhante foi percebido também no estudo de Ramos, Santos e Reis (2013), em que foi realizada uma revisão bibliográfica sobre a EAN em escolares.

Quanto aos objetivos dos artigos selecionados, cerca de $27 \%$ tinham por propósito a intervenção com base na adesão ao tratamento de doenças como a hipertensão arterial sistêmica e o diabetes mellitus tipo 2, que, segundo Reiners et al. (2008), a adesão ao tratamento de tais enfermidades é algo ainda insatisfatório, sendo um desafio constante nos serviços de saúde. Contudo, predominou entre os estudos o objetivo de avaliar a intervenção a partir de seu efeito sobre o grupo escolhido. Dessa forma, entende-se que as metodologias aplicadas estão baseadas somente nos resultados, diminuindo o processo educativo, que é fazer com que a aprendizagem alcance a modificação do comportamento alimentar e o empoderamento em relação a sua saúde (RAMOS; SANTOS; REIS, 2013).

Dada a crescente demanda de indivíduos com algum tipo de DCNT, muitos dos estudos encontrados relacionam suas ações de EAN com grupos que já atingidos com alguma dessas doenças, que são o grande público atendido na APS. Fazem-se então necessárias ações que atendam não somente 
a esse público, mas também intervenções voltadas para a prevenção de doenças e promoção da saúde que sejam permanentes e contextualizadas na realidade dos indivíduos, tornando essencial compreender os conceitos que envolvem a promoção da saúde, uma vez que a EAN baseia-se na promoção da alimentação adequada e saudável como forma de construir ambientes saudáveis, afim de proporcionar uma qualidade de vida melhor à população (BRASIL, 2016C).

Observou-se que, entre o público-alvo dos estudos, predominou, entre os usuários adultos da APS, o sexo feminino; o que, segundo Gomes et al. (2013), justifica-se devido ao fato de as mulheres fazerem mais o uso dos serviços de saúde e do interesse maior de participar de estudos em que são desenvolvidos temas de promoção à saúde. Entre a faixa etária dos participantes, preponderaram usuários maiores de 20 anos, porém ressalta-se a presença de idosos em cerca de $100 \%$ dos estudos, que mesmo não sendo a busca prioritária neste estudo, essa população é pertinente entre aqueles que frequentam a APS.

Segundo Martins et al. (2014), essa presença marcante dos idosos nos serviços de saúde se deve ao envelhecimento populacional no País e o fortalecimento do cuidado à saúde da população idosa que passou a ser uma prioridade no Sistema Único de Saúde. Destacase também a presença de um adolescente em um dos estudos selecionados, porém os autores não discutem a relevância nem a consequência de tal inclusão. Entretanto, sabe-se que intervenções podem e devem ser específicas para as diferentes faixas etárias, a fim de garantir a compreensão e a adesão correta do que será proposto na intervenção.

Várias são as formas de ações educativas que foram utilizadas, bem como a variedade de intervenções aplicadas em um mesmo grupo de estudo, prevalecendo, sobretudo, o uso de palestras e aulas com métodos expositivos, sendo relatada em apenas um dos artigos o uso da problematização como ferramenta para o desenvolvimento das oficinas. Nota-se, portanto, uma forte tendência metodológica clássica aderida aos termos 'aulas expositivas' ou 'palestras dialogadas' percebida de forma relevante nos estudos analisados, além do uso de recursos como as dramatizações, vídeos, fotos e cartazes, entre outros recursos abordados. Segundo Santos (2012), o uso de tais artifícios busca melhorar a interação do grupo com o tema exposto, porém acabam caindo em tipos de técnicas associadas a um modelo de educação tradicional, de transmissão de conhecimentos e verticalidade na relação entre educador e educando.

Encontram-se também intervenções, entre os estudos selecionados, baseadas no modelo do aconselhamento dietético, como oficinas culinárias, visitas domiciliares e com olhar da educação ambiental, como as hortas cultivadas em pequenos espaços. Contudo, em parte dos trabalhos, as intervenções não são descritas em detalhes, apenas citam superficialmente aspectos como o período, os conteúdos abordados e as técnicas utilizadas, corroborando o estudo de Santos (2012), que reforça a predominância de estudos que não descrevem de forma aprofundada as atividades desenvolvidas no processo educativo. Ressalta-se, portanto, a importância de ampliar o uso das ferramentas que incluem a culinária e o fortalecimento da cultura local e familiar, ainda pouco abordados nas práticas descritas.

Diante disso, a EAN deve basear-se na ampliação da autonomia e no fortalecimento da participação ativa dos sujeitos, aumentando a capacidade de escolha dos indivíduos, bem como o de transformar e produzir em sua própria realidade (BRASIL, 2012A). Destaca-se que educar não é adestrar, dessa forma, a transmissão de conhecimento limita as ações da EAN, pois alimentar-se envolve muito mais que ingerir nutrientes necessários a uma boa qualidade de vida, a alimentação compreende um universo de significados que perpassa do prazer pessoal até fatores socioculturais nos quais o indivíduo está inserido. 
Segundo Boog (1997), essa abordagem clássica e prática que instrui o outro a como proceder negligenciando os conflitos e contradições não resulta em uma abordagem eficaz da EAN, devendo então haver uma aproximação mais abrangente no desenvolvimento de novos métodos, a fim de gerar situações de reflexão, com base na problematização e na busca de soluções alternativas, compreendendo toda a singularidade que envolve o indivíduo e sua relação com a alimentação, que, para Santos (2012), convencionou-se chamar de 'experiências exitosas', pois leva o indivíduo a entender e relacionar o problema com a prática real e a mudança possível.

Entende-se que um trabalho multiprofissional compreende o cuidado de um objeto por diferentes disciplinas, não devendo haver desacordos entre as concepções e as estratégias traçadas (ALVARENGA ET AL., 2013). Partindo da compreensão de EAN, como um serviço multiprofissional, intersetorial e transdisciplinar, dos trabalhos analisados, verifica-se que mais de $50 \%$ dos estudos tiveram o envolvimento de outros profissionais para o desenvolvimento do projeto, prevalecendo a presença do educador físico, entre outros profissionais, como farmacêuticos e fisioterapeutas. Assim, percebe-se a importância do trabalho interdisciplinar, em que se caracteriza como uma integração das disciplinas por concepções e métodos, quando se refere à EAN, visto que questões relacionadascom a alimentação tange diversos outros pontos que transcendem a praticidade de só se alimentar; ou relações somente com a nutrição superam a centralização na doença, perpassando pelo desenvolvimento de estratégias que abordem a complexidade pertinente à saúde (FERIOTTI, 2009).

As avaliações das intervenções propostas se deram a partir do conhecimento dos participantes por meio de questionários antes e após a intervenção, partindo do total de números de acertos. Entretanto, apenas um questionário utilizado nos diferentes estudos era validado, o que demonstra a inexistência de padronização e a fragilidade que tais instrumentos podem apresentar, podendo levar à falta de credibilidade da metodologia utilizada. Esse entendimento parte da hipótese questionada por Gazzinelli et al. (2005), de que a apreensão de saber instituído sempre leva à aquisição de novos comportamentos e práticas. No entanto, a simples avaliação do conhecimento adquirido por si só não leva à confirmação de mudança de comportamento alimentar dos sujeitos, é necessária uma avaliação mais crítica da intervenção, partindo da complexidade dos sujeitos, e não se apoiar somente em resultados objetivos.

A antropometria foi também um dos métodos utilizados para a avaliação da intervenção, visto que o conhecimento sobre a alimentação saudável leva a hábitos alimentares mais adequados, acarretando uma melhoria do estado nutricional. Todavia, segundo Friedrich, Schuch e Wagner (2012), intervenções isoladas não promovem mudanças no Índice de Massa Corporal (IMC), e isso pode ser explicado devido às mudanças na massa corporal não ocorrerem em período curto de tempo, sendo necessária uma maior duração na intervenção, sendo que os estudos apresentaram períodos que variaram entre 7 semanas e 15 meses. Assim, a educação nutricional é um processo que demanda tempo para obter resultados positivos; e, como qualquer ação educativa, exige manutenção e continuidade, tornando-se um desafio para os profissionais de saúde.

Dentre os espaços escolhidos para o desenvolvimento das intervenções, além de dispor da Unidade Básica de Saúde (UBS) e da ESF, destaca-se o Programa Academia da Saúde (PAS), que conta com uma infraestrutura, equipamentos e profissionais qualificados para a orientação de práticas corporais, atividade física, lazer e modos de vida saudáveis (BRASIL, 2011). Assim, o uso de tais espaços para a implementação das atividades é visto como um potencializador das ações de cuidados individuais e coletivos na atenção básica, de modo que a melhora nos hábitos 
alimentares, associados à prática de atividade física contribui para a redução do risco de desenvolvimento das doenças crônicas não transmissíveis (TEIXEIRA ET AL., 2013).

A crescente importância da EAN no contexto da promoção da saúde e da alimentação saudável é vista como uma estratégia imprescindível no enfrentamento de novos desafios nos campos da saúde, alimentação e nutrição No entanto, cabe ressaltar as poucas referências sobre o arcabouço teórico, metodológico e operacional, que norteiam as políticas públicas no campo da educação nutricional. Os vazios de ordem conceitual, ideológica e organizacional no desenvolvimento das ações da EAN, referidos na literatura, norteiam a necessidade de pensar nas escolhas das atividades de participação coletiva baseados em metodologias ativas como rodas de conversa, as oficinas e a problematização como estratégias facilitadoras (BOTELHO ET AL., 2016), sendo importante privilegiar o uso de métodos que promovam a participação coletiva, propiciando relatos da realidade e aproximando profissionais e usuários.

Ante essas necessidades, foram publicados, no ano de 2016, pelo Ministério da Saúde e a Universidade Federal de Minas Gerais, trabalhos importantes que trazem essa discussão sobre a urgência de desempenhar ações de promoção da saúde elencadas na autonomia e no empoderamento, bem como que sejam efetivas e contextualizadas na realidade dos sujeitos, ferramentas imprescindíveis para a construção de intervenções educativas pautadas na EAN (BRASIL, 2016A, 2016B, 2016C).

Como não há um padrão de metodologias a serem desenvolvidas nas intervenções de EAN, nem do tamanho amostral nem tampouco variáveis a serem analisadas, isso traz limitações para a discussão dos resultados, pois dificulta a comparação dos métodos desenvolvidos nas intervenções. Dificuldade também levantada nos estudos de Barbosa et al. (2016) e Barros, Saunders e Leal et al. (2008), devido às metodologias diversificadas encontradas nos artigos analisados. Contudo, os estudos trazem resultados positivos quanto às intervenções aplicadas, destacando as mudanças relatadas quanto ao perfil alimentar, bem como do estado nutricional e dos parâmetros bioquímicos, quanto à glicemia e colesterol, com melhora da pressão arterial e no conhecimento sobre alimentação saudável.

Além disso, alguns estudos trazem suas próprias limitações no desenvolvimento das intervenções como dificuldades de logística e infraestrutura, a não alocação aleatória dos grupos, o curto espaço de tempo, o tamanho da amostra, perdas amostrais do grupo, ausência de grupo controle, entre outras, dificuldades estas que podem ser explicadas devido ao local e ao público escolhido para a aplicação das intervenções.

Outras dificuldades que podem ser observadas relacionadas com o cotidiano dos serviços de saúde para o desenvolvimento de intervenções de EAN, limitações estas inerentes ao próprio serviço - como a grande rotatividade dos usuários e de profissionais, o abandono do tratamento, a falta de apoio da gestão para as atividades de educação em saúde -, muitas vezes visam somente à produtividade, e não à qualidade do serviço prestado, ao perfil e ao conhecimento do profissional de saúde sobre a temática, às condições de vida e de pobreza local, fatos estes levantados no estudo de Vasconcelos e Magalhães (2016) como barreiras para a realização de ações de nutrição contínuas e consistentes dentro da ESF, que para superá-las requerem atuações que busquem a melhoria dos serviços em um âmbito macro das políticas públicas de saúde.

\section{Conclusões}

Este estudo demonstra a relevância da EAN para o fortalecimento das ações de nutrição, auxiliando no empoderamento dos indivíduos e no desenvolvimento da 
autonomia diante de suas escolhas alimentares. Entretanto, para que alcancem tais objetivos de forma efetiva, são necessários estudos que descrevam de forma detalhada as técnicas aplicadas, com uso de ferramentas coesas e validadas, a serem empregadas nas diferentes situações.

Existem, atualmente, ferramentas importantes aplicadas à prática da EAN na atenção básica, como o Instrutivo: metodologia de trabalho em grupos para ações de alimentação e nutrição na atenção básica; e Desmistificando dúvidas sobre alimentação e nutrição: material de apoio para profissionais de saúde, que visam à valorização da cultura alimentar dos sujeitos, bem como de sua comunidade e família, e dos alimentos acessíveis em sua localidade. Tais instrumentos reforçam conceitos importantes levantados na EAN, e como alcançá-los na prática da rotina de um serviço de saúde auxiliando os profissionais na tomada de decisão.

Faz-se também necessária uma deliberação política para o desenvolvimento das ações de EAN dentro da APS, com descrição dessas atividades nos planos municipais de saúde e no planejamento das práticas educativas nas unidades, possibilitando o investimento e o apoio gerencial e logístico necessário para o seu efetivo desenvolvimento a fim de garantir não só a realização, mas também a continuidade dessas atividades dentro dos serviços de saúde.

Nessa perspectiva, ressalta-se a urgência de redirecionamento das pesquisas relacionadas com a EAN no âmbito da APS no Brasil, sendo pertinente a necessidade de maiores esforços para o delineamento e a condução de estudos que evidenciem modelos de intervenção com métodos ativos e inovadores de educação em saúde.

\section{Referências}

ALENCAR, A. P. A. et al. Impacto do programa mais médicos na atenção básica de um município do sertão central nordestino. Revista Eletrônica Gestão \& Sociedade, Belo Horizonte, v. 10, n. 26, p. 1290-1301, ago. 2016. Disponível em: <https://www.gestaoesociedade. org/gestaoesociedade/article/viewFile/2085/1166>. Acesso em: 2 nov. 2016.

ALVARENGA, J. P. O. et al. Multiprofissionalidade e interdisciplinaridade na formação em saúde: vivências de graduandos no estágio regional interprofissional. Revista de enfermagem UFPE, Recife, v. 7, n. 1, p. 59445951, out. 2013.
ANUNCIAÇÃO, P. C. et al. Avaliação do conhecimento sobre alimentação antes e após intervenção nutricional entre diabéticos tipo 2. Revista Baiana de Saúde Pública, Salvador, v. 36, n. 4, p. 986-1001, out./dez. 2012.

BARBOSA, L. B. et al. Estudos de avaliação do conhecimento nutricional de adultos: uma revisão sistemática. Ciência \& Saúde Coletiva, Rio de Janeiro, v. 21, n. 2, p. 449-462, fev. 2016. Disponível em: <http://www.scielo. $\mathrm{br} / \mathrm{pdf} / \mathrm{csc} / \mathrm{v} 21 \mathrm{n} 2 / 1413-8123-\mathrm{csc}-21-02-0449 . \mathrm{pdf}>$. Acesso em: 2 nov. 2016.

BARROS, D. C.; SAUNDERS, C.; LEAL, M. C. Avaliação nutricional antropométrica de gestantes 
brasileiras: uma revisão sistemática. Revista Brasileira

de Saúde Materno Infantil, Recife, v. 8, n. 4, p. 363-376, dez.2008. Disponível em: <http://www. scielo.br/scielo.php?script=sci_arttext\&pid =S1519-38292008000400002>. Acesso em: 2 nov. 2016.

BOOG, M. C. F. Educação em Nutrição: integrando experiências. Campinas: Komedi, 2013.

Educação nutricional: passado, presente, futuro. Revista de Nutrição: PUCCAMP, Campinas, v. 10, n. 1, p. 5-19, 1997. Disponível em: <https://www.faculdadeguararapes.edu.br/site/hotsites/biblioteca/educacaonutricional_passado-presente-futuro59500.pdf>. Acesso em 2 nov. 2016.

BOTELHO, F. C. et al. Estratégias pedagógicas em grupos com o tema alimentação e nutrição: os bastidores do processo de escolha. Ciência \& Saúde Coletiva, Rio de Janeiro, v. 21, n. 6, p. 1889-1898, jun. 2016. Disponível em: <http://www.scielo.br/scielo.php?pid=S1413$-81232016000601889 \&$ script $=$ sci_abstract\&tlng $=\mathrm{pt}>$. Acesso em: 2 nov. 2016

BRASIL. Ministério da Saúde. Avaliação de Efetividade de Programas de Atividade Física no Brasil. Brasília, DF: Ministério da Saúde, 2011.

Ministério da Saúde. Desmistificando dúvidas sobre alimentação e nutrição: material de apoio para profissionais de saúde. Brasília, DF: Ministério da Saúde, 2016a.

Ministério da Saúde. Na cozinha com as frutas, legumes e verduras. Brasília, DF: Ministério da Saúde, $2016 b$.

Ministério da Saúde. Secretaria de Atenção à Saúde. Departamento de Atenção Básica. Política Nacional de Atenção Básica. Brasília, DF: Ministério da Saúde, 2012a.

. Ministério da Saúde; UNIVERSIDADE

FEDERAL DE MINAS GERAIS. Instrutivo: metodologia de trabalho em grupos para ações de alimentação e nutrição na atenção básica. Brasília, DF: Ministério da Saúde, 2016c.
Ministério do Desenvolvimento Social e

Combate à Fome. Secretaria Nacional de Segurança

Alimentar e Nutricional. Marco de Referência de

Educação Alimentar e Nutricional para as Políticas

Públicas. Brasília, DF: Ministério da Saúde, 2012b.

DEUS, R. M. et al. Impacto de intervenção nutricional sobre o perfil alimentar e antropométrico de usuárias do Programa academia da saúde. Ciência \& Saúde Coletiva, Rio de Janeiro, v. 20, n. 6, p. 1937-1946, jun. 2015 .

FERIOTTI, M. L. Equipe multiprofissional, transdisciplinaridade e saúde: desafios do nosso tempo. Vínculo, São Paulo, v. 6, n. 2, p. 179-190, dez. 2009. Disponível em: <http://pepsic.bvsalud.org/scielo.php?script=sci_ar ttext\&pid=S1806-24902009000200007 $>$. Acesso em: 2 nov. 2016.

FRIEDRICH, R. R.; SCHUCH, I.; WAGNER, M. B. Efeito de intervenções sobre o índice de massa corporal em escolares. Revista de Saúde Pública, São Paulo, v. 46, n. 3, p. 551-560, jun. 2012. Disponível em: <http:// www.scielo.br/scielo.php?script=sci_arttext\&pid =S0034-89102012000300018>. Acesso em: 2 nov. 2016.

GANDRA, F. P. P. et al. Efeito de um programa de educação no nível de conhecimento e nas atitudes sobre o diabetes mellitus. Revista Brasileira de Promoção em Saúde, Fortaleza, v. 24, n. 4, p. 322-331, out./dez. 2011. Disponível em: <http://periodicos.unifor.br/RBPS/article/view/2089>. Acesso em: 2 nov. 2016.

GAZZINELLI, F. G. et al. Educação em saúde: conhecimentos, representações sociais e experiências da doença. Cadernos de Saúde Pública. Rio de Janeiro, v. 21 n. 1 , fev. 2005.

GOMES, A. C. M. et al. Impacto de estratégias de educação nutricional sobre variáveis antropométricas e conhecimento alimentar. Revista Brasileira de Promoção em Saúde, Fortaleza, v. 26, n. 4, p. 462-469, out./dez. 2013. Disponível em: <http://periodicos.unifor.br/ RBPS/article/view/3111>. Acesso em: 2 nov. 2016.

GREENWOOD, S. A.; FONSECA, A. B. Espaços e caminhos da educação alimentar e nutricional no livro 
didático. Ciência e Educação. Bauru, v. 22, n. 1, p. 201218, 2016. Disponível em: <http://www.scielo.br/pdf/ ciedu/v22n1/1516-7313-ciedu-22-01-0201.pdf>. Acesso em: 2 nov. 2016.

JAIME, P. C. et al. Ações de alimentação e nutrição na atenção básica: a experiência de organização no Governo Brasileiro. Revista de Nutrição, Campinas, v. 24, n. 6, p. 809-824, dez. 2011. Disponível em: $<$ http://www.scielo.br/scielo.php?pid=S1415$-52732011000600002 \&$ script $=$ sci_abstract\&tlng=pt $>$. Acesso em: 2 nov. 2016.

MACHADO, J. C. et al. Análise de três estratégias de educação em saúde para portadores de hipertensão arterial. Ciência \&t Saúde Coletiva, Rio de Janeiro, v. 21, n. 2, p. 611-620, fev. 2016. Disponível em: <http://www.scielo.br/scielo.php?pid=S1413$-81232016000200611 \&$ script=sci_abstract\&tlng=pt $>$. Acesso em: 2 nov. 2016.

MARTINS, A. B. et al. Atenção Primária à Saúde voltada as necessidades dos idosos: da teoria à prática. Ciência \&t Saúde Coletiva, Rio de Janeiro, v. 19, n. 8, p. 3403-3416, ago. 2014. Disponível em: <http://www.scielo.br/scielo.php?pid=S1413$-81232014000803403 \&$ script=sci_abstract $\&$ tlng $=p t>$. Acesso em: 2 nov. 2016.

MENDONÇA, R. D.; LOPES, A. C. S. Efeitos de intervenções em saúde sobre os hábitos alimentares e medidas físicas. Revista da Escola de Enfermagem da USP, São Paulo, v. 46, n. 3, p. 573-579, jun. 2012. Disponível em: <http://www.revistas.usp.br/reeusp/article/ view/40983>. Acesso em: 2 nov. 2016.

OLIVEIRA, M. A. C.; PEREIRA, I. C. Atributos essenciais da Atenção Primária e a Estratégia Saúde da Família. Revisa Brasileira de Enfermagem, Brasília, DF, v. 66, n. esp, p. 158-164, set. 2013. Disponível em: $<$ http://www.scielo.br/pdf/reben/v66nspe/v66nspea20.pdf>. Acesso em: 2 nov. 2016.

PIMENTEL, I. R. S. et al. Caracterização da demanda em uma Unidade de Saúde da Família. Revista Brasileira de Medicina de Família e Comunidade, Florianópolis, p. 175-81, jul./set. 2011. Disponível em: <https://www.rbmfc.org.br/rbmfc/article/download/95/364>. Acesso em: 2 nov. 2016.

RAMOS, F. P.; SANTOS, L. A. S.; REIS, A. B. C.

Educação alimentar e nutricional em escolares: uma revisão de literatura. Cadernos de Saúde Pública, Rio de Janeiro, v. 29, n. 11, p. 2147-2161, nov. 2013. Disponível em: <http://www.cookie.com.br/site/wp-content/ uploads/2015/05/Educa\%C3\%A7\%C3\%A3o-alimentare-Nutricional-em-Escolares-.pdf $>$. Acesso em: 2 nov. 2016.

REINERS, A. A. O. et al. Produção bibliográfica sobre adesão/não-adesão de pessoas ao tratamento de saúde. Ciência \&t Saúde Coletiva, Rio de Janeiro, v. 13, supl. 2, p. 2299-2306, dez. 2008. Disponível em: <http://www.scielo.br/scielo.php?pid=S1413$-81232008000900034 \&$ script $=$ sci $\_$abstract $\&$ tlng $=p t>$. Acesso em: 2 nov. 2016.

RIBEIRO, A. G. et al. Hipertensão arterial e orientação domiciliar: o papel estratégico da saúde da família. Revista de Nutrição, Campinas, v. 25, n. 2, p. 271-282, mar./abr. 2012. Disponível em: <http:// www.scielo.br/scielo.php?script=sci_arttext\&pid =S1415-52732012000200009>. Acesso em: 2 nov. 2016.

SANTOS, L. A. S. O fazer educação alimentar e nutricional: algumas contribuições para reflexão. Ciência \&̇ Saúde Coletiva, Rio de Janeiro, v. 17, n. 2, p. 453-462, fev. 2012. Disponível em: <http:// www.scielo.br/scielo.php?script=sci_arttext\&pid $=$ S1413-81232012000200018>. Acesso em: 2 nov. 2016.

SILVA, C. P. et al. Intervenção nutricional pautada na estratégia de oficinas em um serviço de promoção da saúde de Belo Horizonte, Minas Gerais. Revista de Nutrição, Campinas, v. 26, n. 6, p. 647-658, nov./dez. 2013. Disponível em: <http:// www.scielo.br/scielo.php?script=sci_arttext\&pid =S1415-52732013000600004>. Acesso em: 2 nov. 2016.

SILVA, R. A. et al. Intervenção nutricional integrada à prática de Lian Gong em usuários hipertensos de uma unidade básica de saúde de Belo Horizonte, Minas Gerais. Revista APS, Juiz de Fora, v. 15, n. 3, p. 356-363, jul./set. 2012. 
SILVA, T. A. P.; QUINTÃO, D. F. Estratégias de educação nutricional nos grupos do projeto 'de bem com a balança' de 4 unidades básicas de saúde do município de Muriaé - MG. Revista Brasileira de Obesidade, Nutrição e Emagrecimento, São Paulo, v. 9, n. 53, p. 188-198, set./out. 2015. Disponível em: <www.rbone. com.br/index.php/rbone/article/download/392/361>. Acesso em: 2 nov. 2016.

TEIXEIRA, P. D. S. et al. Intervenção nutricional educativa como ferramenta eficaz para mudança de hábitos alimentares e peso corporal entre praticantes de atividade física. Ciência \&t Saúde Coletiva, Rio de Janeiro, v. 18, n. 2, p. 347-356,fev. 2013. Disponível em: $<$ http://www.scielo.br/scielo.php?script=sci_arttext\&pi d=S1413-81232013000200006 >. Acesso em: 2 nov. 2016.
VASCONCELOS, A. C. C. P.; MAGALHAES, R. Práticas educativas em Segurança Alimentar e Nutricional: reflexões a partir da experiência da Estratégia Saúde da Família em João Pessoa, PB, Brasil. Interface: Comunicação, Saúde e Educação, Botucatu, v. 20, n. 56, p. 99-110, mar. 2016. Disponível em: <http:// www.scielo.br/pdf/icse/v20n56/1807-5762-icse-1807-576220150156.pdf>. Acesso em: 2 nov. 2016.

Recebido para publicação em dezembro de 2016 Versão final em junho de 2017 Conflito de interesses: inexistente

Suporte financeiro: não houve 